\title{
A Case Report of Water Intoxication During Radioactive lodine Treatment: Why Physicians Should Communicate Clearly With Patients
}

\author{
Samuel Teye a, b, c, Nico Malan ${ }^{\mathrm{a}, \mathrm{c}}$, Mboyo-di-Tamba Vangu ${ }^{\mathrm{a}, \mathrm{c}, \mathrm{d}}$
}

\begin{abstract}
Radioactive iodine (RAI) treatment is an effective method for the treatment of thyroid remnant ablation and metastasis in patients with differentiated thyroid cancer. The current guidelines recommend patients to drink lots of water to reduce the amount of iodine in the body during RAI treatment; however, water intoxication is a life-threatening condition resulting from hyponatremia. This case report describes water intoxication during RAI treatment in a 55-year-old patient who was evaluated for the management of angio-invasive follicular thyroid cancer following a total thyroidectomy. Hyponatremia is an electrolyte imbalance commonly encountered in oncology practice and is usually defined as a serum sodium level of less than $135 \mathrm{mEq} / \mathrm{L}$. Water intoxication is a rare phenomenon that occurs due to an excessive intake of water, especially when the volume of water intake exceeds the excretory capacity of the kidney. This case report has revealed that all relevant information and instructions including the consumption of water should be clearly and accurately communicated to patients.
\end{abstract}

Keywords: Water intoxication; Hyponatremia; Radioactive treatment; Differentiated thyroid carcinoma

\section{Introduction}

Hyponatremia is an electrolyte imbalance commonly encountered in oncology practice and is usually defined as a serum

Manuscript submitted May 5, 2020, accepted May 22, 2020

Published online August 26, 2020

aDepartment of Nuclear Medicine and Molecular Imaging, Faculty of Health Sciences, University of the Witwatersrand/Charlotte Maxeke Johannesburg Academic Hospital, Johannesburg, South Africa

bInternational Atomic Energy Agency, Vienna, Austria

${ }^{\mathrm{c}}$ These authors contributed equally to this work.

${ }^{\mathrm{d} C}$ Corresponding Author: Mboyo-di-Tamba Vangu, Department of Nuclear Medicine and Molecular Imaging, Faculty of Health Sciences, University of the Witwatersrand/Charlotte Maxeke Johannesburg Academic Hospital, 7 York Road, Parktown, Johannesburg, South Africa.

Email: Mboyo-di-tamba.vangu@wits.ac.za

doi: https://doi.org/10.14740/jem646 sodium level of less than $135 \mathrm{mEq} / \mathrm{L}[1,2]$. Water intoxication is a rare phenomenon that occurs due to an excessive intake of water, especially when the volume of water intake exceeds the excretory capacity of the kidney [3]. This results in the reduction in the concentration of serum sodium concentration and hence hyponatremia ensues. Water intoxication is usually observed in psychiatric patients, child-abuse victims and iatrogenic causes [4]. Self-induced water intoxication is known to psychologists, but there is a paucity of information and little awareness of this life-threatening problem in the medical literature [4].

Radioactive iodine (RAI) treatment is an effective method for the treatment of thyroid remnant ablation and metastasis in patients with differentiated thyroid cancer (DTC). Patients are encouraged to increase water intake to assist with the clearance of RAI; however, when this is excessive, resultant hyponatremia may be life-threatening. This case report highlights that instructions to patients, including the intake of water, should be communicated clearly and precisely.

\section{Case Report}

A 55-year-old female was evaluated for the management of angio-invasive follicular thyroid cancer following a total thyroidectomy. She had no known medical condition and was not on any prescription medicine. She underwent an iodine-131 (I-131) diagnostic total body scan after administration of $74 \mathrm{MBq}$. Her thyroid stimulating hormone (TSH) was ideal for imaging and measured $65 \mathrm{mIU} / \mathrm{L}$ at the time. The study showed two foci of uptake in the neck, consistent with remnant thyroid tissue. There was also normal biodistribution of tracer in the salivary glands, stomach, intestines and urinary bladder (Fig. 1). No other areas of abnormal uptake were noted. Single photon emission tomography/computed tomography (SPECT/ CT) images localized the uptake of tracer in the thyroid bed, right side more than the left side (Fig. 2).

The patient was clinically staged as cT3aNoMo, stage II (AJCC 8th edition). She was categorized as an intermediate risk patient for recurrence and/or persistent disease. The patient was counselled regarding the need for further management with RAI treatment to reduce the risk of recurrence. She was also counselled with regards to general radiation protection measures and encouraged to drink ample water and void 


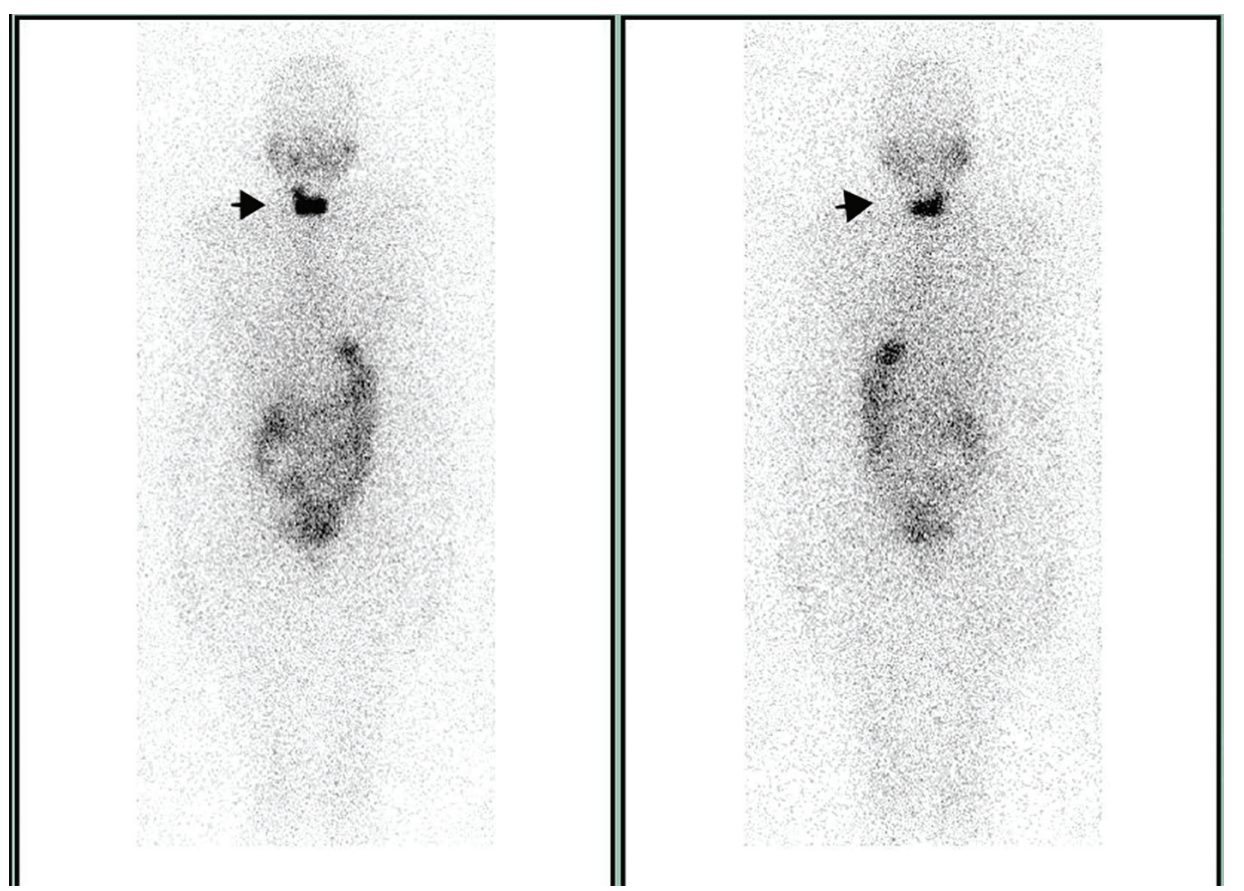

Figure 1. The $74 \mathrm{MBq}$ scan with $\mathrm{l}-131$ showing two foci of uptake in the neck, consistent with remnant thyroid tissue (indicated by arrow).

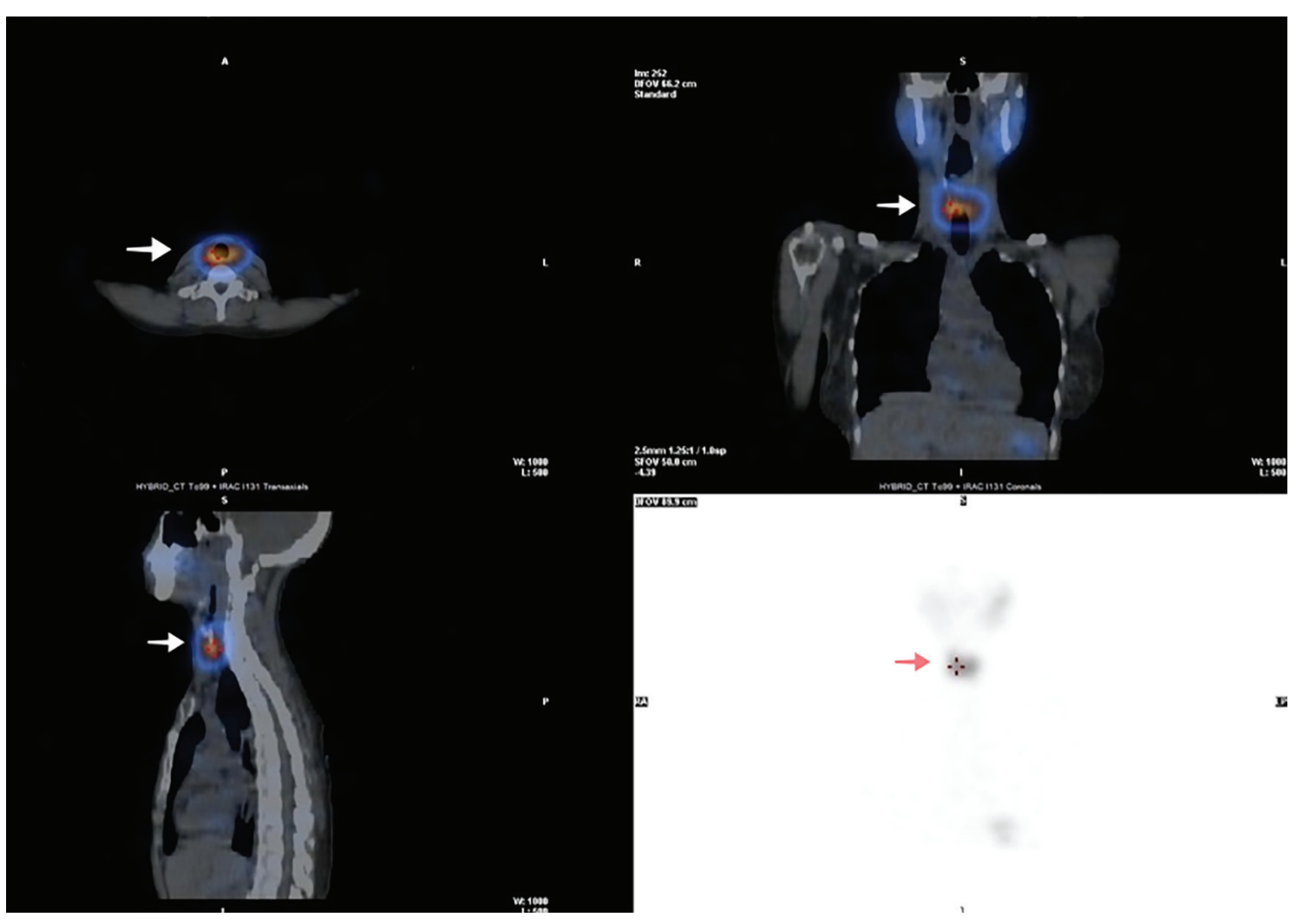

Figure 2. SPECT/CT reconstructed images of the neck and thorax (following $74 \mathrm{MBq}$ of I-131) showing uptake of tracer in the thyroid bed, right side more than the left side (indicated by arrow). SPECT/CT: single photon emission tomography/computed tomography. 
frequently to encourage the clearance of RAI. The patient consented to being treated and was subsequently admitted for treatment. Baseline blood prior to RAI administration (done 3 months prior to admission) was unremarkable as shown in Table 1. Upon admission, the patient's vital signs were normal and the natural stimulated TSH, following total thyroidectomy was adequately raised to allow treatment with RAI (blood pressure: 108/67 $\mathrm{mm} \mathrm{Hg}$; heart rate: $82 \mathrm{bpm}$; temperature: $36.4{ }^{\circ} \mathrm{C}$; respiratory rate: $18 / \mathrm{min}$ ). Two weeks prior to admission to the hospital, she was placed on low iodine diet. The 370 MBq I-131 was administered as an oral capsule as per departmental protocol. The dose rate after administration was 110 $\mu \mathrm{Sv} / \mathrm{h}$. The patient started to complain of nausea after about 24 $\mathrm{h}$ following admission. This was initially attributed to possible gastritis. This was then followed by complaints of some muscle cramps thereafter. The patient was treated symptomatically with analgesics (paracetamol $500 \mathrm{mg}$ qid; metoclopramide 10 $\mathrm{mg}$ bid and pantoprazole $20 \mathrm{mg}$ daily).

Thirty-six hours following admission, the patient developed a generalized seizure which was reported to the attending doctor by nursing staff. Upon examination the patient was confused and drowsy, but still responsive. We arranged for urgent basic blood investigations (Table 1), urgent CT scan of the brain (Fig. 3) and a neurological consultation. The presumptive working diagnosis included a possible metastatic brain lesion that was causing the symptoms. Whilst waiting for the urgent CT brain, the patient developed another generalized seizure, and was throughout drowsy and confused. She was started on anti-seizure medication ( $2 \mathrm{mg}$ intravenous clonazepam as necessary and $400 \mathrm{mg}$ sodium valproate orally 12 hourly) in the interim to prevent subsequent seizures. The urgent $\mathrm{CT}$ brain was generally unremarkable and only revealed a small calcified granuloma (approximately $5 \mathrm{~mm}$ ) in the left occipital lobe of the brain (Fig. 3). The baseline and blood results following the symptoms are tabulated in Table 1.

Based on the blood results, the working diagnosis was changed to that of acute hyponatremia as a possible cause for her symptoms (Table 1). In retrospect, this information ties in with history given by the patient when she initially complained of nausea and muscle cramps (when she was still lucid) and responded to the registrar's enquiry that she consumed $8 \mathrm{~L}$ of water (eight jugs of water, volume of the jug equals $1 \mathrm{~L}$ ) over a relative short period of less than $24 \mathrm{~h}$. Upon further enquiry she said that she consumed this amount of water since she was instructed to "take a lot of water to eliminate the radioactive material from her body". The patient was immediately transferred to intensive care unit (ICU) (dose rate was $23 \mu \mathrm{Sv} / \mathrm{h}$ and considered safe for transfer to a non-isolated ward). A nasogastric tube and urinary catheter were inserted. She was still drowsy and confused. Vital signs at this point in time were also normal. Fluid was restricted and a slow 5\% hypertonic saline intravenous infusion was started. Her vital signs were monitored closely until she regained consciousness $48 \mathrm{~h}$ after her admission to ICU. Her urine and blood osmolality were corrected over $48 \mathrm{~h}$. On the third day of admission to ICU, she was referred back to the general ward where she stayed for an additional 2 days for observation and then discharged home. Prior to her discharge, the therapy scan done was identical to her diagnostic scan.

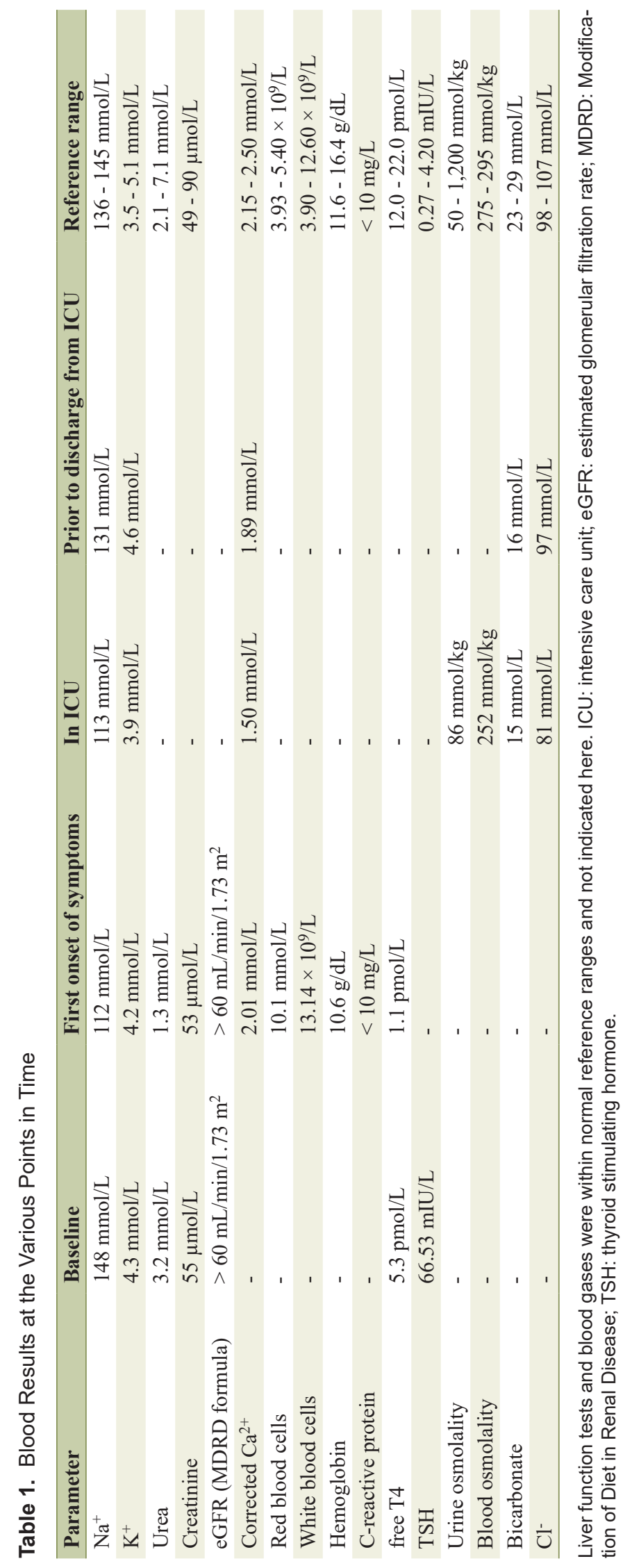




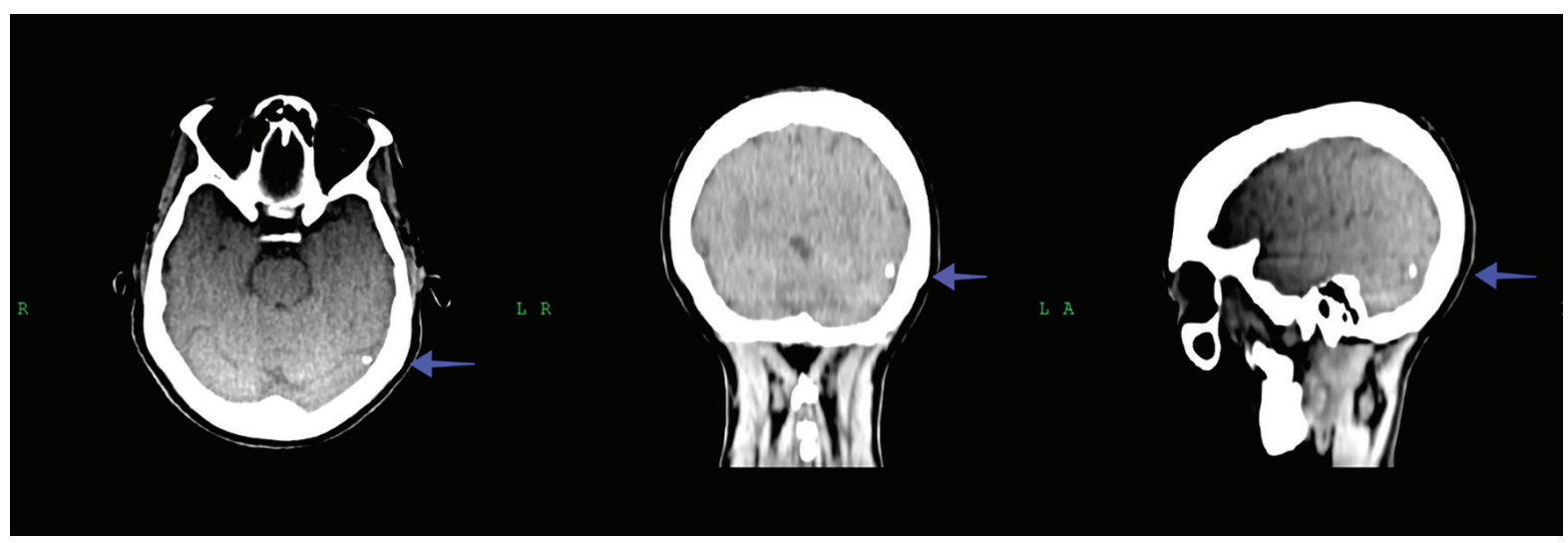

Figure 3. CT scan of the brain showing the small focus of calcification in the left occipital lobe of the brain (indicated by arrow). CT: computed tomography.

The mild hypocalcemia and low free $\mathrm{T} 4$ which may cause related symptoms, were not suspected as potential culprits as the patient improved clinically and was asymptomatic following the correction of the sodium. Also, while we did not measure serum adrenocorticotropic hormone (ACTH) and cortisol level, decreased urinary and blood osmolality and rapid improvement of symptoms by intravenous hypertonic infusion suggest the development of water intoxication in our patient.

We would like to caution: acute hyponatremia from excessive water intoxication as a result of vague patient instruction, and possible fear of radiation may be fatal!

\section{Discussion}

In neurosurgical and neurocritical care settings, there has been a report of $50 \%$ and $38 \%$ incidence of hyponatremia respectively [5, 6] and an overall mortality of $38 \%$ [7]. The hypothalamus, which is found in the brain is essential in controlling body water. We become thirsty when more than $0.5 \%$ of the body water is lost in various ways [8]. The kidneys are capable of removing about $20-28 \mathrm{~L}$ of water a day to control water load and hence excessive water ingestion rarely causes hyponatremia [8]. However, a decrease in serum sodium inhibits antidiuretic hormone (ADH) secretion by the posterior pituitary gland and results in an increase in excretion of water by the kidneys. Hyponatremia develops only when the water intake exceeds the water excretory capacity of the kidneys [810]. The maximum amount of water that a healthy renal system can tolerate is $0.8-1 \mathrm{~L} / \mathrm{h}$ to avoid symptoms of hyponatremia [3]. In hyponatremia, water moves from the extracellular fluid (ECF) to the intracellular fluid (ICF) space to maintain osmolality at a consistent level. Cellular edema occurs in all tissue in hyponatremia; however, cellular edema of the brain is more dangerous than other tissue because the brain is confined within the skull [11].

Under physiological conditions, brain osmolality is in equilibrium with ECF osmolality. In hyponatremia, water moves into the brain in response to the osmotic gradient leading to cerebral edema. Glial cells (astrocytes) that form the blood-brain barrier are mostly affected with sparing of the neurons [12].
Water moves through aquaporin receptors type 1 and 4 into the brain. Brain swelling activates the $\mathrm{Na}^{+}-\mathrm{K}^{+}$ATPase pump to extrude $\mathrm{Na}^{+}$. Other active ions such as $\mathrm{Ca}^{+}$and $\mathrm{K}^{+}$are also extruded through their various channels. Secondly, there is a loss of organic osmolyte (e.g., glutamate, taurine and glycine) and myoinositol through putative volume-sensitive organic osmolyte and anion channels [13, 14]. Loss of organic osmolyte, particularly glutamate which is a neuroactive process, produces transient neurological abnormalities, such as seizures.

Early clinical features of hyponatremia include nausea, vomiting and anorexia. Advanced features may include: headaches, muscular cramps, weakness, lethargy, restlessness, psychosis, depressed reflexes and seizures. Advanced features include coma and respiratory arrest $[3,7]$. In diagnosing hyponatremia, a careful and thorough history and physical examination is essential. Important laboratory investigations should include serum osmolality, urine osmolality, urine $\mathrm{Na}^{+}$ concentration, thyroid function test and glucocorticoid hormone levels [2]. Imaging investigations may include a chest $\mathrm{X}$-ray and CT scan of the brain or abdomen according to the clinical symptoms. Hyponatremia is a medical emergency and prompt treatment is vital as it may cause cerebral edema, cerebral herniation and death. Hypertonic saline $(3-5 \% \mathrm{NaCl})$ is usually used in symptomatic hyponatremia. During administration, the level of the electrolytes should be monitored every $2 \mathrm{~h}$. Correction of electrolytes must be done slowly in order not to exceed the brain's ability to recapture lost osmolality which could result in an inverse osmotic gradient leading to dehydration of the brain and possible demyelination of the white matter [13]. The serum $\mathrm{Na}^{+}$level should not be corrected by 12 $\mathrm{mEq} / \mathrm{L} / 12 \mathrm{~h}$ or more [3]. Patients undergoing RAI treatment for differentiated thyroid carcinoma are routinely encouraged to ingest about $2-3 \mathrm{~L}$ ( 8 - 10 cups) of water a day following treatment with RAI, to encourage diuresis and thus enhancing the elimination of RAI through renal excretion [15].

Even though some guidelines recommend drinking ample water to assist in the elimination of radioactivity on the basis of enhanced diuresis, there is however no large body of evidencebased literature to support this [16-18]. In fact, Haghighatafshar et al [18] showed that although inadequate fluid intake and dehydration may reduce the excretion of iodine, increas- 
ing fluid intake and urinary flow above the physiological range does not significantly increase excretion of RAI. Factors such as baseline serum iodine, volume of remnant functioning thyroid tissue and the presence of a hypothyroid state in thyroidectomized patients may play a role and impact on the physiological excretion of RAI [18, 19].

\section{Conclusion}

This case study indicates clearly that any information, including the consumption of water should not merely be vaguely communicated to the patient, but accurate and clear instructions should be given, that overhydration may be lethal and highlight the need for tailored evidence-based instructions with regards hydration of a patient that is being treated with RAI.

\section{Acknowledgments}

The authors acknowledge the Department of Nuclear Medicine and Molecular Imaging, Charlotte Maxeke Johannesburg Academic Hospital/University of the Witwatersrand.

\section{Financial Disclosure}

This case report was self-funded.

\section{Conflict of Interest}

The authors declare no conflict of interest for the case presented.

\section{Informed Consent}

The patient consented to this case report.

\section{Author Contributions}

The manuscript was conceived and drafted by ST and NM, all figures and tables were created by NM. MTV reviewed the manuscript and made valuable suggestions.

\section{Data Availability}

The data supporting the findings of this case report are available from the corresponding author upon reasonable request.

\section{References}

1. Palmer BF, Gates JR, Lader M. Causes and management of hyponatremia. Ann Pharmacother. 2003;37(11):16941702.

2. Berardi R, Caramanti M, Castagnani M, Guglielmi S, Marcucci F, Savini A, Morgese F, et al. Hyponatremia is a predictor of hospital length and cost of stay and outcome in cancer patients. Support Care Cancer. 2015;23(10):30953101.

3. Annals of pediatric endocrinology \& metabolism [Internet]. 2019. Available from: https://e-apem.org/.

4. Joo MA, Kim EY. Hyponatremia caused by excessive intake of water as a form of child abuse. Ann Pediatr Endocrinol Metab. 2013;18(2):95-98.

5. Upadhyay A, Jaber BL, Madias NE. Incidence and prevalence of hyponatremia. Am J Med. 2006;119(7 Suppl 1):S30-35.

6. Kirkman MA, Albert AF, Ibrahim A, Doberenz D. Hyponatremia and brain injury: historical and contemporary perspectives. Neurocrit Care. 2013;18(3):406-416.

7. Ayus JC, Achinger SG, Arieff A. Brain cell volume regulation in hyponatremia: role of sex, age, vasopressin, and hypoxia. Am J Physiol Renal Physiol. 2008;295(3):F619624.

8. Cahyanur R, Sarwono J, Armelia L, Marbun MBH, Soewondo P. Non-psychogenic polydipsia in 45-year-old man with primary hyperparathyroidism and recurrent bilateral nephrolithiasis. Med J Indones. 2012;21:230-234.

9. Schulman J. Infantile water intoxication at home. Pediatrics. 1980;66(1):119-120.

10. Bruce RC, Kliegman RM. Hyponatremic seizures secondary to oral water intoxication in infancy: association with commercial bottled drinking water. Pediatrics. 1997;100(6):E4.

11. Radojevic N, Bjelogrlic B, Aleksic V, Rancic N, Samardzic M, Petkovic S, Savic S. Forensic aspects of water intoxication: four case reports and review of relevant literature. Forensic Sci Int. 2012;220(1-3):1-5.

12. Kimelberg HK. Water homeostasis in the brain: basic concepts. Neuroscience. 2004;129(4):851-860.

13. h t t p : / / u n m f m. p b w o r k s. c o m / f / hyponatremia+review+NEJM.pdf. 2019.

14. Fisher SK, Heacock AM, Keep RF, Foster DJ. Receptor regulation of osmolyte homeostasis in neural cells. J Physiol. 2010;588(Pt 18):3355-3364.

15. Ziessman HA, O'Malley JP, Thrall JH. Nuclear medicine: the requisites e-book. Elsevier Health Sciences. 2013.

16. Bushberg JT, Boone JM. The essential physics of medical imaging. Lippincott Williams \& Wilkins; 2011.

17. Nmz H. Surface contamination in skin and room during hospitalization of thyroid cancer patient receiving radioiodine ablation. IOSR J Dent Med Sci. 2012;2:27-33.

18. Haghighatafshar M, Banani A, Zeinali-Rafsanjani B, Etemadi Z, Ghaedian T. Impact of the amount of liquid intake on the dose rate of patients treated with radioiodine. Indian J Nucl Med. 2018;33(1):10-13.

19. Cheen Hoe AK, Fong LY, Halim FNA, Fatt QK, Hamzah F. The minimum amount of fluids needed to achieve the fastest time to reach permissible level for release in well-differentiated thyroid patients undergoing high-dose I-131 therapy. World J Nucl Med. 2018;17(3):182-187. 